\title{
(C) OPEN ACCESS \\ Dexamethasone and dexmedetomidine as adjuvants to local anesthetic mixture in intercostal nerve block for thoracoscopic pneumonectomy: a prospective randomized study
}

\author{
Panpan Zhang, ${ }^{1}$ Shijiang Liu, ${ }^{2}$ Jingming Zhu, ${ }^{2}$ Zhuqing Rao, ${ }^{2}$ Cunming Liu ${ }^{2}$
}

\begin{abstract}
'Department of Anesthesiology, The Affiliated Suzhou Hospital of Nanjing Medical University, Suzhou, China

${ }^{2}$ Department of Anesthesiology, The First Affiliated Hospital of Nanjing Medical University(Jiangsu Province Hospital), Nanjing, China
\end{abstract}

Correspondence to Dr Cunming Liu and Dr Zhuqing Rao, Department of Anesthesiology, The First Affiliated Hospital of Nanjing Medical University (Jiangsu Province Hospital), Nanjing, 210029, China; 1335587409@qq.com, zhuqingrao@njmu.edu.cn

PZ and SL contributed equally. $\mathrm{ZR}$ and $\mathrm{CL}$ contributed equally.

Received 31 October 2018 Revised 17 July 2019 Accepted 24 July 2019 Published Online First 8 August 2019

\begin{abstract}
Background and objectives Perineural

dexamethasone or dexmedetomidine prolongs the duration of single-injection peripheral nerve block when added to the local anesthetic solution. In a randomized, controlled, double-blinded study in patients undergoing thoracoscopic pneumonectomy, we tested the hypothesis that combined perineural dexamethasone and dexmedetomidine prolonged the duration of analgesia as compared with either perineural dexamethasone or perineural dexmedetomidine after intercostal nerve block (INB).
\end{abstract}

Methods Eighty patients were randomized to receive INB using $28 \mathrm{~mL} 0.5 \%$ ropivacaine, with $2 \mathrm{~mL}$ normal saline (R group), with $10 \mathrm{mg}$ dexamethasone in $2 \mathrm{~mL}$ (RS group) or $1 \mu \mathrm{g} / \mathrm{kg}$ dexmedetomidine in $2 \mathrm{~mL}$ (RM group), or with $1 \mu \mathrm{g} / \mathrm{kg}$ dexmedetomidine and $10 \mathrm{mg}$ dexamethasone in $2 \mathrm{~mL}$ (RSM group) administrated perineurally. The INB was performed by the surgeon under thoracoscopic direct vision; a total of six intercostal spaces were involved, each with an injection of $5 \mathrm{~mL}$. The primary outcome was the duration of analgesia. Secondary outcomes included total postoperative fentanyl consumption, visual analog scale pain score and safety assessment (adverse effects).

Results The duration of analgesia in RSM (824.2 $\pm 105.1 \mathrm{~min}$ ) was longer than that in RS (611.5 $\pm 133.0 \mathrm{~min}), \mathrm{RM}(602.5 \pm 108.5 \mathrm{~min})$ and $R$ $(440.0 \pm 109.6 \mathrm{~min})(p<0.001)$. Total postoperative fentanyl consumption was lower in RSM $(106.0 \pm 84.0 \mu \mathrm{g})$ compared with RS $(243.0 \pm 175.2 \mu \mathrm{g})$, RM

$(237.0 \pm 98.7 \mu \mathrm{g})$ and $R(369.0 \pm 134.2 \mu \mathrm{g})(\mathrm{p}<0.001)$. No significant difference was observed in the incidences of adverse effects between the four groups.

Conclusion The addition of combined perineural dexmedetomidine and dexamethasone to ropivacaine for INB seemed to be an attractive method for prolonged analgesia with almost no adverse effects.

Trial registration number ChiCTR-IOR-17012183.

\section{INTRODUCTION}

Single-injection peripheral nerve block (PNB) is (C) American Society of Regional Anesthesia \& Pain Medicine 2019. Re-use permitted under CC BY-NC. No commercial re-use. Published by BMJ.

To cite: Zhang P, Liu S, Zhu J, et al. Reg Anesth Pain Med 2019:44:917-922. commonly used for perioperative analgesia and anesthesia. ${ }^{1}$ Although PNB are beneficial for improved early postoperative pain management, it is often insufficient, as postoperative pain can persist for several days. The aim of prolonging the duration of PNB to treat postoperative pain is a key issue in regional anesthesia. Three approaches for extending the duration of PNB include continuous PNB with catheter-based techniques, novel local anesthetics delivery systems and addition of novel adjuvants to local anesthetics. ${ }^{2}$

Adjuvants that are frequently added to local anesthetics to prolong analgesia following single-injection PNB include epinephrine, opioids, tramadol, ketamine, midazolam, magnesium, clonidine, dexmedetomidine and dexamethasone, but often with limited success and unproven safety. ${ }^{3-6}$ Studies of perineural buprenorphine, dexamethasone and dexmedetomidine have most consistently demonstrated prolongation of PNB. ${ }^{5}$

Dexamethasone is a potent long-acting steroid that has shown efficacy as an adjuvant to local anesthetics in various studies. ${ }^{7}$ Dexmedetomidine enhances PNB when added to local anesthetics, providing better quality of anesthesia as well as postoperative analgesia. ${ }^{9} 10$ The mechanism by which dexamethasone and dexmedetomidine prolong the duration of local anesthetics are not completely understood and may arise from various factors. Both dexamethasone and dexmedetomidine can reduce local inflammation and prolong the duration of nerve block through vasoconstriction by maintaining the local concentration of the local anesthetic. ${ }^{11-14}$ Vasoconstriction also inhibits the nociceptive impulse transmission along myelinated C fibers. ${ }^{15}$ Possible mechanisms of dexmedetomidine in prolonging the duration of nerve blocks may also include the inhibition of the hyperpolarization-activated cation current (Ih current). ${ }^{16}$ Some research suggests that dexmedetomidine may provide local anesthetic action that blocks the conduction of nerve signals through $\mathrm{C}$ and $\mathrm{A}_{\delta}$ fibers, not through $\alpha_{2}$ action, and may stimulate the release of enkephalin-like substances at peripheral sites. $^{17}$

Due to the different mechanisms of action, we hypothesized that ropivacaine combined with both perineural dexamethasone and dexmedetomidine could further prolong the duration of PNB. Therefore, we performed a prospective, randomized, double-blinded, controlled trial to investigate whether the addition of two adjuvants (dexmedetomidine and dexamethasone) to ropivacaine for INB prolongs block duration and therefore improves postoperative analgesia, as compared with each adjuvant alone with ropivacaine. 


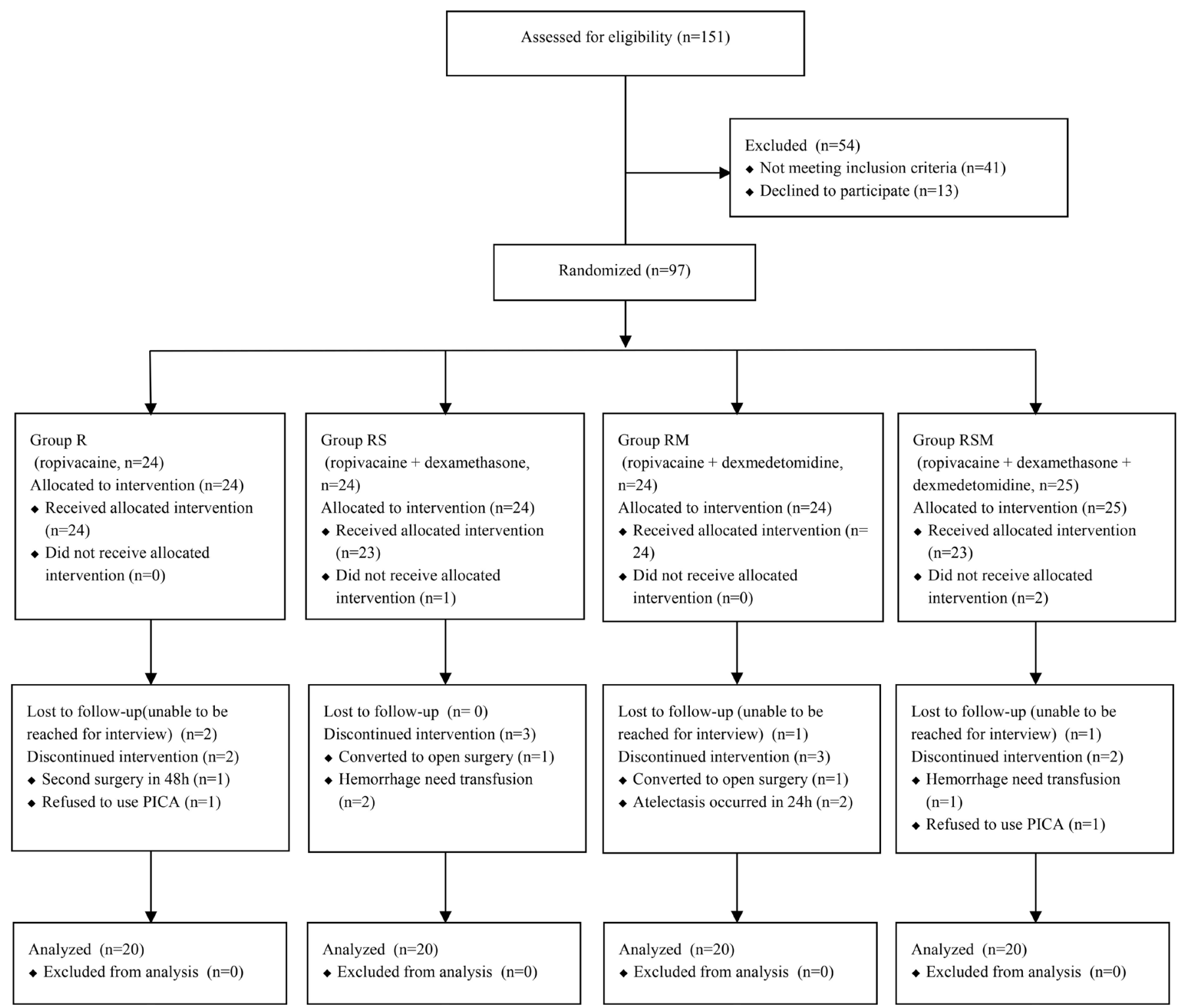

Figure 1 Patient enrollment, inclusion and exclusion process. PICA, patient-controlled intravenous analgesia.

\section{METHODS}

\section{Trial design and participants}

After providing written informed consent, patients with American Society of Anesthesiologists (ASA) physical status classes I and II, aged 28-68 years and scheduled for thoracoscopic

\begin{tabular}{lcccc}
\hline Table 1 & \multicolumn{5}{c}{ Demographic data and surgical characteristics } \\
\hline & Group R & Group RS & Group RM & Group RSM \\
\hline Age (years) & $53.3 \pm 7.8$ & $54.5 \pm 9.3$ & $53.9 \pm 6.7$ & $55.6 \pm 10.3$ \\
\hline BMI (kg/m $\left.\mathrm{m}^{2}\right)$ & $23.6 \pm 2.0$ & $22.8 \pm 2.5$ & $22.1 \pm 2.4$ & $23.5 \pm 2.1$ \\
Gender (F/M) & $9 / 11$ & $12 / 8$ & $13 / 7$ & $14 / 6$ \\
$\begin{array}{l}\text { Duration of } \\
\text { surgery (min) }\end{array}$ & $132.4 \pm 51.9$ & $126.7 \pm 19.4$ & $119.6 \pm 49.7$ & $136.4 \pm 29.7$ \\
$\begin{array}{l}\text { Extubation } \\
\text { time (min) }\end{array}$ & $19.5 \pm 11.2$ & $22.2 \pm 6.3$ & $25.8 \pm 9.5$ & $23.7 \pm 10.1$ \\
\hline
\end{tabular}

Data are expressed as means $\pm \mathrm{SD}$ or numbers. There were no significant differences between groups.

$B M I$, body mass index; F, female; $M$, male; $R$, ropivacaine; $R M$,

ropivacaine+dexmedetomidine; $\mathrm{RS}$, ropivacaine+dexamethasone; RSM,

ropivacaine+dexamethasone+dexmedetomidine. pneumonectomy between September 2017 and April 2018 were considered eligible to participate in this prospective, randomized, double-blinded clinical trial. Major exclusion criteria were: hypertension; ischemic heart disease; psychiatric disorders; preoperative heart rate (HR) less than 50 beats/min with/ without cardiac conduction or rhythm abnormalities; diabetes mellitus; neuromuscular and endocrine diseases; coagulation disorders; adrenoreceptor agonist or antagonist therapy; chronic pain treatment or chronic steroid therapy; allergy to the study drugs and refusal to participate in the study. Individuals who had an estimated intraoperative blood loss of more than $500 \mathrm{~mL}$ were subsequently excluded from the study. Those who were transferred to an open chest operation, those who required a second operation for postoperative hemorrhage and those who presented severe postoperative infection were also subsequently excluded from the study.

\section{Randomization and blinding}

A computer-generated random numbers list was used to randomize consented study participants on a $1: 1: 1: 1$ ratio to receive INB with trial medication. 
Table 2 Duration of analgesia and total postoperative fentanyl consumption

\begin{tabular}{|c|c|c|c|c|c|}
\hline Variable & Group R & Group RS & Group RM & Group RSM & $\mathbf{P}$ \\
\hline Duration of analgesia (min) & $440.0 \pm 109.6$ & $611.5 \pm 133.0$ & $602.5 \pm 108.5$ & $824.2 \pm 105.1$ & $<0.001$ \\
\hline Total postoperative fentanyl consumption $(\mu \mathrm{g})$ & $369.0 \pm 134.2$ & $243.0 \pm 175.2$ & $237.0 \pm 98.7$ & $106.0 \pm 84.0$ & $<0.001$ \\
\hline
\end{tabular}

Duration of analgesia and total postoperative fentanyl consumption data are expressed as means \pm SD.

$R$, ropivacaine; $R M$, ropivacaine+dexmedetomidine; $R S$, ropivacaine+dexamethasone; $R S M$, ropivacaine+dexamethasone+dexmedetomidine.

1. Group $\mathrm{R}$ (control): $28 \mathrm{~mL}$ of ropivacaine $0.5 \%+2 \mathrm{~mL}$ normal saline.

2. Group RS: $28 \mathrm{~mL}$ of ropivacaine $0.5 \%+$ perineural dexamethasone $10 \mathrm{mg}$ in $2 \mathrm{~mL}$.

3. Group RM: $28 \mathrm{~mL}$ of ropivacaine $0.5 \%+$ perineural dexmedetomidine $1 \mu \mathrm{g} / \mathrm{kg}$ in $2 \mathrm{~mL}$.

4. Group RSM: $28 \mathrm{~mL}$ of ropivacaine $0.5 \%+$ perineural dexamethasone $10 \mathrm{mg}$ and perineural dexmedetomidine $1 \mu \mathrm{g} / \mathrm{kg}$ in $2 \mathrm{~mL}$.

The volume of study drug was always $30 \mathrm{~mL}$. All patients were not administered intravenous dexmedetomidine or dexamethasone perioperatively. Their patient-controlled intravenous analgesia (PCIA) protocol was programmed with $1 \mathrm{mg}$ fentanyl diluted to $100 \mathrm{~mL}$ ( $3 \mathrm{~mL}$ bolus, lockout time interval of $8 \mathrm{~min}$ and 1 hour limit of $15 \mathrm{~mL}$ without any baseline infusion). The randomization table was kept in the hospital pharmacy. The study medication and PCIA solutions were prepared by a nurse who did not participate in the study. The patients, surgeon and research staff who enrolled patients and collected study data were blinded to group assignment.

\section{Interventions}

Before the study procedure, all the patients were trained how to use the PCIA pump and also instructed on using a $10 \mathrm{~cm}$ visual analog scale (VAS; with 0: no pain, to 10: the worst imaginable pain).

On arrival in the operating room, an 18-gage intravenous cannula was inserted into a peripheral vein on the arm, and an infusion of lactated Ringer's solution was started. Five-lead ECG, HR, invasive blood pressure, respiratory rate (RR) and pulse oxygen saturation $\left(\mathrm{SpO}_{2}\right)$ were continuously monitored. General anesthesia was induced with $0.08-0.10 \mathrm{mg} / \mathrm{kg}$ of midazolam, $0.15-0.30 \mathrm{mg} / \mathrm{kg}$ of etomidate, $2-4 \mu \mathrm{g} / \mathrm{kg}$ of fentanyl and $0.12 \mathrm{mg} / \mathrm{kg}$ of cisatracurium. Maintenance of anesthesia was achieved with propofol, sevoflurane, remifentanil and atracurium.

At the end of the surgery, the INB was performed by the surgeon under thoracoscopic direct vision. At the site of the surgical incision (generally three incisions, usually between ribs 4-8) and including the adjacent upper and lower two intercostal spaces, a total of six intercostal spaces were involved, each with an injection of $5 \mathrm{~mL}$. All patients received PCIA for postoperative analgesia. PCIA was administered for VAS $\geq 4$ or on patient request.

\section{Outcomes}

Duration of analgesia, defined as time in minutes to the first request for analgesic, was designated as the primary outcome. Total postoperative fentanyl consumption was also recorded within the 48-hour period. In the postanesthesia care unit, time of tracheal extubation was recorded. The patients' HR, mean arterial pressure (MAP), VAS at rest and Ramsay Sedation Score (RSS) (1: anxious patient; 2: cooperative and tranquil; 3 : responding to command; 4 : brisk response to stimulus; 5: sluggish response to stimulus; 6: no response to stimulus) were assessed before surgery (baseline) and at 6 hours, 12 hours, 24 hours, 36 hours and 48 hours after surgery.

Side effects such as bradycardia (HR $<50$ beats/min), hypoxemia $\left(\mathrm{SpO}_{2}<90 \%\right)$, hypotension (systolic blood pressure $<90 \mathrm{mmHg}$ or more than $20 \%$ decline from the baseline), respiratory depression $(\mathrm{RR}<10$ beats/min lasting for more than $10 \mathrm{~min}$ ), headache, pruritus, nausea, vomiting and neurotoxicity were recorded after surgery, whereas hypotension was treated with fluid loading, intravenous ephedrine or phenylephrine. In addition, bradycardia was treated with atropine. Respiratory depression was treated with oxygen or naloxone until $R R \geq 15$ beats/min.

\section{Statistical analysis}

Duration of analgesia was the primary outcome. We hypothesized that perineural dexamethasone and dexmedetomidine combined (RSM group) prolonged the duration of analgesia compared with single adjuvant groups of perineural dexamethasone (RS group) or perineural dexmedetomidine (RM group) after INB. In our preliminary study conducted with eight patients (two in each group), the mean duration of analgesia was $500 \pm 160 \mathrm{~min}$, $570 \pm 226 \mathrm{~min}, 550 \pm 147 \mathrm{~min}, 767 \pm 184 \mathrm{~min}$ in groups R, RS, RM and RSM. Sample size calculation was performed with PASS V.11.0 (PASS, NCSS, USA) for Windows. One-way analysis of variance (ANOVA) was selected and grouped into four groups, group allocation ratios were equal, hypothesized means were 570, 550 and 767 and SD were 226, 147 and 184, respectively. At a power of 0.80 and an alpha error of 0.05 , the required sample size for each group was calculated to be 15 . Considering the dropouts and incomplete follow-up, suggested 20 patients per group and a total of 80 patients for this study.

Statistical analysis was performed with SPSS V.24.0 for Windows and GraphPad Prism 6 (GraphPad Software, La Jolla, California, USA). For duration of analgesia, total postoperative fentanyl consumption and VAS data, ANOVA followed by the Bonferroni post hoc test was used. For timeto-event outcomes, we analyzed data using the Kaplan-Meier survival method and compared groups using the log-rank test, with adjustment for multiple comparisons. Repeated measures ANOVA with Bonferroni correction was used for MAP and HR data. Categorical data (the incidence of side effects) were presented as frequencies (\%) and analyzed using Fisher's exact test. $\mathrm{P}<0.05$ value was considered to be statistically significant.

\section{RESULTS}

A total of 151 patients were approached to participate in this study from September 2017 to April 2018; 54 patients were ineligible due to not meeting inclusion criteria or refusal to participate; 17 patients were excluded because of non-adherence to the protocol (three patients refused to participate in the study after surgery; two patients converted to open surgery; three patients hemorrhage need transfusion; four patients lost to follow-up; one patient underwent second surgery in 48 hours; two patients refused to use PICA after surgery; and two patients 


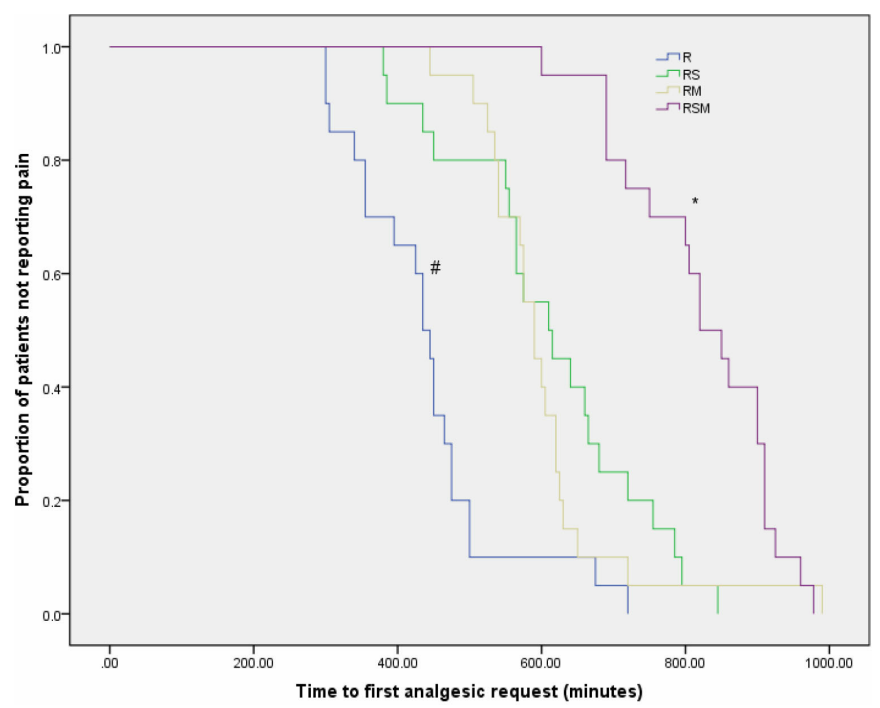

Figure 2 Kaplan-Meier survival plot representing the first analgesic request in the four groups. ${ }^{*} \mathrm{P}<0.001$ between $\mathrm{RSM}$ and other groups. ${ }^{\#} \mathrm{P}<0.001$ between $\mathrm{RS}, \mathrm{RM}$ and $\mathrm{R}$ group. $\mathrm{R}$, ropivacaine; $\mathrm{RM}$, ropivacaine+dexmedetomidine; $R S$, ropivacaine+dexamethasone; RSM, ropivacaine+dexamethasone+dexmedetomidine.

developed actelectasis in 24 hours); and finally, 80 patients were randomized and completed the study protocol (R group: $n=20$; RS group: $n=20$; RM group: $n=20$; and RSM group: $n=20$ ). Figure 1 represents the Consolidated Standards of Reporting Trials flow diagram depicting patient progress through the study phases.

A total of 80 patients were included for analysis in the study. No significant differences in patient characteristics were evident between each group (table 1).

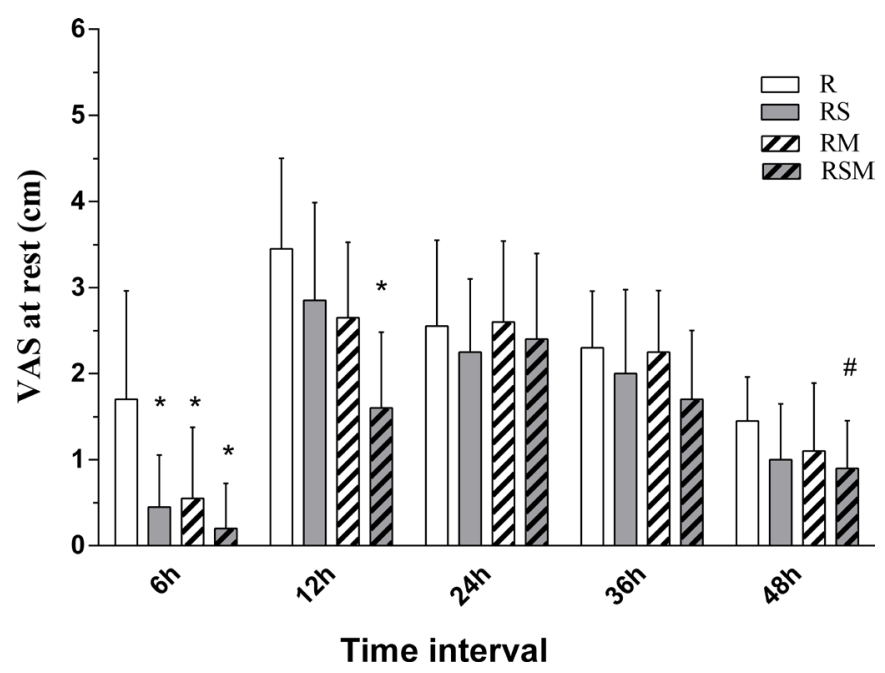

Figure 3 Postoperative pain severity VAS score at rest (in $\mathrm{cm})$ at $6,12,24,36$ and 48 hours postoperatively. At 6 hours postoperatively, ${ }^{*} p<0.001$ group R compared with groups RS, RM and RSM. At 12 hours postoperatively, ${ }^{*} p<0.001$ group RSM compared with groups RS, RM and R. At 48 hours postoperatively, ${ }^{\#} \mathrm{p}=0.043$ between groups RSM and R. $R$, ropivacaine; RM, ropivacaine+dexmedetomidine; $\mathrm{RS}$, ropivacaine+dexamethasone; RSM, ropivacaine+dexamethasone+dexmedetomidine; VAS, visual analog scale.

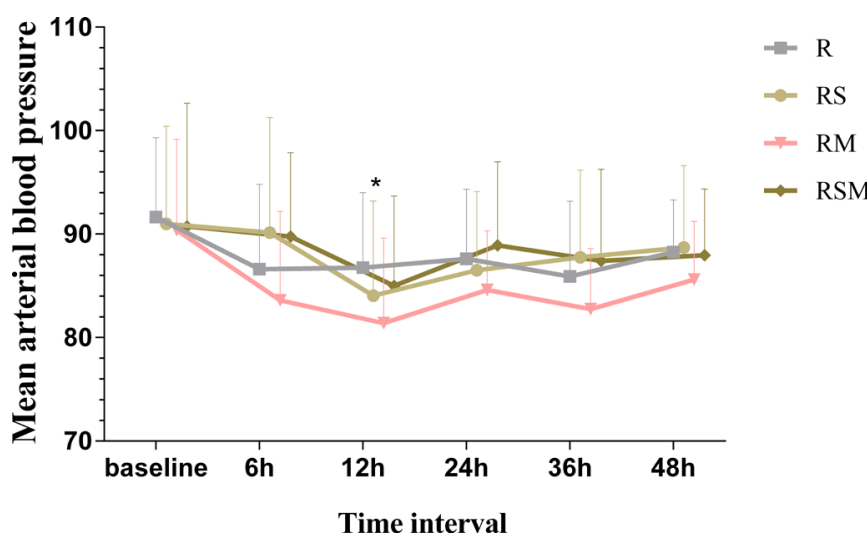

Figure 4 MAP changes at different times in each group. In all groups, the changes in MAP showed a significant difference in relation to time with ${ }^{*} p<0.001$. MAP, mean arterial blood pressure; $R$, ropivacaine; $R M$, ropivacaine+dexmedetomidine; $R S$, ropivacaine+dexamethasone; $R S M$, ropivacaine+dexamethasone+dexmedetomidine.

The duration of analgesia was significantly longer in group RSM compared with the other three groups $(\mathrm{p}<0.001)$. Compared with group RS and group RM, the duration of analgesia was significantly shorter in group $R(p<0.001)$. There was no significant difference between group RS and group RM $(p=1.000)$ (table 2). The Kaplan-Meier survival analysis of the time to the first analgesic request was shown in figure 2 . The log-rank test also suggested prolongation of the first analgesic request for group RSM compared with the other three groups $(\mathrm{p}<0.001)$. Compared with group RS and group RM, the first analgesic request was significantly shorter in group $R(p<0.001)$. There was no significant difference between group RS and group RM $(\mathrm{p}=1.000)$.

Total postoperative fentanyl consumption in group RSM was lower than that the other three groups $(p<0.001)$. Compared with group RS and group RM, total postoperative fentanyl consumption was significantly higher in group $R(p=0.016$ and $\mathrm{p}=0.010$, respectively). There was no significant difference between group RS and group RM ( $\mathrm{p}=1.000)$ (table 2 ).

At 6 hours postoperatively, VAS in group $\mathrm{R}$ was higher than those in the other three groups $(\mathrm{p}<0.001)$. At 12 hours postoperatively, VAS in group RSM was lower compared with group $\mathrm{RS}$, group RM and group R $(\mathrm{p}<0.001)$. At 48 hours postoperatively, VAS was lower in group RSM compared with group $\mathrm{R}(\mathrm{p}=0.043)$. There were no significant differences in VAS among the four groups at 24 hours and 36 hours postoperatively $(\mathrm{p}=0.645$ and $\mathrm{p}=0.076$, respectively) (figure 3 ).

The discrepancies of MAP and HR acquired at different time points were statistically significant $(P<0.001)$. No significant differences were observed between the study groups $(\mathrm{P}=0.242$ and $\mathrm{P}=0.792$, respectively) (figures 4 and 5$)$.

The number of patients experiencing side effects was reported in table 3. No significant differences were observed in the incidences of hypotension, hypoxemia, respiratory depression, vomiting, nausea, pruritus or dizziness among the four groups. The RSS was two in all patients.

\section{DISCUSSION}

In this study, we demonstrated that ropivacaine combined with two perineural adjuvants (dexamethasone and dexmedetomidine) can further prolong the duration of analgesia and reduce the consumption of fentanyl compared with ropivacaine combined with a single perineural adjuvant (dexamethasone 


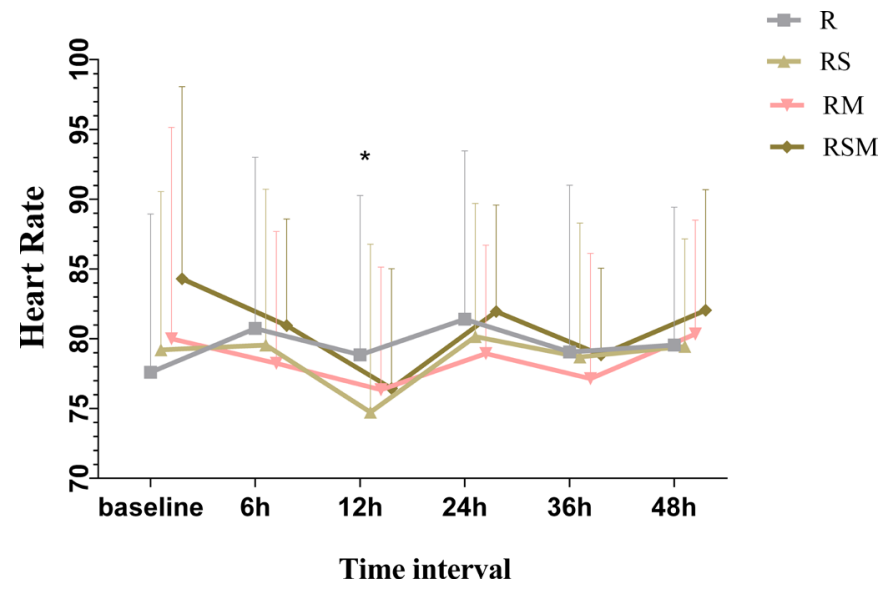

Figure 5 Heart rate changes at different times in each group. In all groups, the changes in HR showed a significant difference in relation to time with ${ }^{*} p<0.001$. $H R$, heart rate; $R$, ropivacaine; $R M$, ropivacaine+dexmedetomidine; $R S$, ropivacaine+dexamethasone; RSM, ropivacaine+dexamethasone+dexmedetomidine.

or dexmedetomidine) or ropivacaine alone in patients undergoing thoracoscopic pneumonectomy with almost no adverse effects.

Although there are multiple adjuvants that could extend the duration of local anesthetics, it was worth noting that the ability of adjuvants to prolong nerve block may have a ceiling. ${ }^{18}$ Similar effect might not have been achieved by simply increasing the dosage of dexamethasone or dexmedetomidine. In the context of that knowledge, we raised the question: could the combination of two adjuvants further prolong the duration of nerve block? From this study, we found that dual agent combination perineural adjuvant drugs increased the duration of effect of ropivacaine almost twofold. This interesting observation was in accordance with the work of Hassan and colleagues, ${ }^{19}$ who observed that the combination of both dexamethasone and dexmedetomidine could further prolonged the sensory block of the caudal block. As local anesthetic adjuvants, dexamethasone and dexmedetomidine might have synergistic or additive effects. Unfortunately, our study has the same flaws as the above study (no intravenous control group), which made it impossible to determine whether the effect was due to systemic or local effects.

Thakur et $a l^{20}$ reported that $1 \mu \mathrm{g} / \mathrm{kg}$ dexmedetomidine was nearly the ideal dose as an adjuvant in axillary blocks and that this

\begin{tabular}{lccccc}
\hline \multicolumn{7}{l}{ Table 3} & \multicolumn{5}{l}{ Incidence of side effects } \\
\hline Side effects & Group R & Group RS & Group RM & Group RSM & P \\
\hline Bradycardia & $0(0)$ & $0(0)$ & $0(0)$ & $0(0)$ & \\
Hypotension & $0(0)$ & $0(0)$ & $0(0)$ & $0(0)$ & \\
Hypoxemia & $0(0)$ & $0(0)$ & $0(0)$ & $0(0)$ & \\
$\begin{array}{l}\text { Respiratory } \\
\text { depression }\end{array}$ & $0(0)$ & $0(0)$ & $0(0)$ & $0(0)$ & \\
Nausea & $0(0)$ & $1(5)$ & $2(10)$ & $2(10)$ & 0.747 \\
Vomiting & $0(0)$ & $2(10)$ & $0(0)$ & $1(5)$ & 0.611 \\
Pruritus & $0(0)$ & $0(0)$ & $0(0)$ & $0(0)$ & \\
Dizziness & $0(0)$ & $1(5)$ & $1(5)$ & $0(0)$ & 1.000 \\
Neurotoxicity & $0(0)$ & $0(0)$ & $0(0)$ & $0(0)$ & \\
\hline
\end{tabular}

Incidence of side effects data are expressed as number and percentage.

$R$, ropivacaine; $R M$, ropivacaine+dexmedetomidine;

$\mathrm{RS}$, ropivacaine+dexamethasone; RSM,

ropivacaine+dexamethasone+dexmedetomidine. dose increases the duration of postoperative analgesia and delays the requirement of the first dose of analgesic. Although dexamethasone was widely used clinically, the optimal dose of dexamethasone as a local anesthetic adjuvant was inconsistent. Previous studies have shown that dexamethasone demonstrated significant beneficial dose-dependent effects as an adjuvant for local anesthetic. ${ }^{21}$ Recent studies have shown that perineural high doses of dexamethasone have no advantage, and low doses of dexamethasone are advocated. ${ }^{22}$ A recent meta-analysis showed very low quality evidence that there was a ceiling effect of dexamethasone at $4 \mathrm{mg} .{ }^{23}$ However, the latest research findings that both intravenous and perineural administered dexamethasone $4 \mathrm{mg}$ has no clinically relevant effect on the duration of sensory block provided by ropivacaine applied to the ulnar nerve in volunteers. ${ }^{24}$ In addition, in the current study, we used a higher dose of dexamethasone than other experts have recommended. Although no permanent complications associated with nerve block could be attributed to the use of dexamethasone, considering that high doses of dexamethasone may increase potential risks, we no longer recommend high doses of dexamethasone administered perineurally. ${ }^{25}$ Therefore, the effective dose of dexamethasone as an adjuvant for local anesthesia needs further study.

There are a number of limitations associated with the present study. First, this study lacks an intravenous control. It would have required more than four groups by adding the intravenous group, making calculations and statistical analysis of the sample size more difficult. However, without intravenous control, we cannot ascertain whether the addition of either dexamethasone or dexmedetomidine was via systemic absorption or through perineural action. Second, we used a higher dose of dexamethasone than other experts have recommended. Third, VAS scores were measured only at rest, thus this reduces the usefulness of the results provided. Fourth, we did not assess the duration of the sensory block in patients using repeated neurological examinations. Considering that frequent testing of sensory block after surgery can affect patients' rest, the time from the end of INB to the first use of fentanyl after operation indicates the duration of analgesia. Fifth, dexamethasone and dexmedetomidine are used off-label. Even though it is widely used on an international level and has been investigated in many scientific trials, the US Food and Drug Administration does not approve dexamethasone or dexmedetomidine for perineural administration. Regarding the inconclusive data on the safety of perineural dexamethasone and dexmedetomidine, we would like to remind clinicians that this 'off-label' use needs approval of the appropriate regulatory health institutions. Finally, our calculation of the number of patients who were needed in our study might not adequate, so the study power cannot differentiate statistically significant differences between the dexamethasone and dexmedetomidine. Of course, further research is needed to confirm this.

\section{CONCLUSIONS}

We demonstrated that patients undergoing thoracoscopic pneumonectomy with the addition of perineural $10 \mathrm{mg}$ dexamethasone and $1 \mu \mathrm{g} / \mathrm{kg}$ dexmedetomidine to $0.5 \%$ ropivacaine for INB had further increases in the duration of analgesia and decreases in postoperative fentanyl consumption compared with patients who did not receive this combination of drugs.

Acknowledgements The authors thank Min Yu, PhD, and Youli Hu, PhD (Department of Anesthesiology, The First Affiliated Hospital of Nanjing Medical University, Nanjing, Jiangsu Province, China) for their critical review of the manuscript. The authors would like to thank all the members of Thoracic Surgery 
Department (The First Affiliated Hospital of Nanjing Medical University, Nanjing, Jiangsu Province, China) for their great help and support.

Contributors All authors helped design the study. PZ, ZR and CL helped conduct of the study. PZ, SL, ZR and CL helped recruit the patients. JZ, ZR and CL helped collect the data. PZ, SL, ZR and CL helped analyze the data. PZ, SL and CL was a major contributor in writing the manuscript. All authors read and approved the final manuscript.

Funding The authors have not declared a specific grant for this research from any funding agency in the public, commercial or not-for-profit sectors.

Competing interests None declared.

Patient consent for publication Not required.

Ethics approval The study protocol was approved by the Ethics Committee of The First Affiliated Hospital of Nanjing Medical University (Nanjing, Jiangsu Province, China) and was prospectively registered on Chinese Clinical Trial Registry (registration number ChiCTR-IOR-17012183)

Provenance and peer review Not commissioned; externally peer reviewed. Data availability statement Data are available on reasonable request.

Open access This is an open access article distributed in accordance with the Creative Commons Attribution Non Commercial (CC BY-NC 4.0) license, which permits others to distribute, remix, adapt, build upon this work non-commercially, and license their derivative works on different terms, provided the original work is properly cited, an indication of whether changes were made, and the use is noncommercial. See: http://creativecommons.org/licenses/by-nc/4.0/.

\section{REFERENCES}

1 Fredrickson Fanzca MJ, Danesh-Clough TK, White R. Adjuvant dexamethasone for bupivacaine sciatic and ankle blocks: results from 2 randomized placebo-controlled trials. Reg Anesth Pain Med 2013;38:300-7.

2 King CH, Beutler SS, Kaye AD, et al. Pharmacologic properties of novel local anesthetic agents in anesthesia practice. Anesthesio/ Clin 2017:35:315-25.

3 Bailard NS, Ortiz J, Flores RA. Additives to local anesthetics for peripheral nerve blocks: evidence, limitations, and recommendations. Am J Health Syst Pharm 2014;71:373-85

4 Swain A, Nag DS, Sahu S, et al. Adjuvants to local anesthetics: current understanding and future trends. World J Clin Cases 2017;5:307-23.

5 Kirksey MA, Haskins SC, Cheng J, et al. Local anesthetic peripheral nerve block adjuvants for prolongation of analgesia: a systematic qualitative review. PLOS One 2015; 10:e0137312.

6 Knight JB, Schott NJ, Kentor ML, et al. Neurotoxicity of common peripheral nerve block adjuvants. Curr Opin Anaesthesiol 2015;28:598-604.

7 Bjørn S, Linde F, Nielsen KK, et al. Effect of perineural dexamethasone on the duration of single injection saphenous nerve block for analgesia after major ankle surgery: a randomized, controlled study. Reg Anesth Pain Med 2017:42:210-6.

8 Parrington SJ, O'Donnell D, Chan VWS, et al. Dexamethasone added to mepivacaine prolongs the duration of analgesia after supraclavicular brachial plexus blockade. Reg Anesth Pain Med 2010;35:422-6.

9 Fritsch G, Danninger T, Allerberger K, et al. Dexmedetomidine added to ropivacaine extends the duration of interscalene brachial plexus blocks for elective shoulder surgery when compared with ropivacaine alone: a single-center, prospective, tripleblind, randomized controlled trial. Reg Anesth Pain Med 2014;39:37-47

10 Hussain N, Grzywacz VP, Ferreri CA, et al. Investigating the efficacy of dexmedetomidine as an adjuvant to local anesthesia in brachial plexus block: a systematic review and meta-analysis of 18 randomized controlled trials. Reg Anesth Pain Med 2017:42:184-96.

$11 \mathrm{Hu}$ J, Vacas S, Feng $X$, et al. Dexmedetomidine prevents cognitive decline by enhancing resolution of high mobility group box 1 protein-induced inflammation through a Vagomimetic action in mice. Anesthesiology 2018;128:921-31.

12 Dräger C, Benziger D, Gao F, et al. Prolonged intercostal nerve blockade in sheep using controlled-release of bupivacaine and dexamethasone from polymer microspheres. Anesthesiology 1998;89:969-79.

13 Yabuki A, Higuchi $H$, Yoshitomi T, et al. Locally injected dexmedetomidine induces vasoconstriction via peripheral $\alpha-2 A$ adrenoceptor subtype in guinea pigs. Reg Anesth Pain Med 2014;39:133-6

14 Marks R, Barlow JW, Funder JW. Steroid-Induced vasoconstriction: glucocorticoid antagonist studies. J Clin Endocrinol Metab 1982;54:1075-7.

15 Johansson A, Hao J, Sjölund B. Local corticosteroid application blocks transmission in normal nociceptive C-fibres. Acta Anaesthesiol Scand 1990;34:335-8.

16 Brummett CM, Hong EK, Janda AM, et al. Perineural dexmedetomidine added to ropivacaine for sciatic nerve block in rats prolongs the duration of analgesia by blocking the hyperpolarization-activated cation current. Anesthesiology 2011:115:836-43.

17 Nakamura M, Ferreira SH. Peripheral analgesic action of clonidine: mediation by release of endogenous enkephalin-like substances. Eur J Pharmacol 1988;146:223-8.

18 Rwei AY, Sherburne RT, Zurakowski D, et al. Prolonged duration local anesthesia using liposomal bupivacaine combined with liposomal dexamethasone and dexmedetomidine. Anesth Analg 2018;126:1170-5.

19 Hassan PF, Hassan AS, Elmetwally SA. Caudal analgesia for hypospadias in pediatrics: comparative evaluation of adjuvants dexamethasone and dexmedetomidine combination versus dexamethasone or dexmedetomidine to bupivacaine: prospective, double-blinded, randomized comparative study. Anesth Essays Res 2018:12:644-50.

20 Thakur A, Singh J, Kumar S, et al. Efficacy of dexmedetomidine in two different doses as an adjuvant to lignocaine in patients scheduled for surgeries under axillary block. $J$ Clin Diagn Res 2017;11:16-21.

21 Woo JH, Kim YJ, Kim DY, et al. Dose-dependency of dexamethasone on the analgesic effect of interscalene block for arthroscopic shoulder surgery using ropivacaine $0.5 \%$ : A randomised controlled trial. Eur J Anaesthesiol 2015;32:650-5.

22 Bravo D, Aliste J, Layera S, et al. A multicenter, randomized comparison between 2, 5, and $8 \mathrm{Mg}$ of perineural dexamethasone for ultrasound-guided infraclavicular block. Reg Anesth Pain Med 2019:44:46-51.

23 Kirkham KR, Jacot-Guillarmod A, Albrecht E. Optimal dose of perineural dexamethasone to prolong analgesia after brachial plexus blockade: a systematic review and meta-analysis. Anesth Analg 2018;126:270-9.

24 Marhofer P, Columb M, Hopkins PM, et al. Dexamethasone as an adjuvant for peripheral nerve blockade: a randomised, triple-blinded crossover study in volunteers. Br J Anaesth 2019:122:525-31.

25 Williams BA, Hough KA, Tsui BYK, et al. Neurotoxicity of adjuvants used in perineural anesthesia and analgesia in comparison with ropivacaine. Reg Anesth Pain Med 2011;36:225-30. 